\title{
The Unmet Data Visualization Needs of Decision Makers within Organizations
}

\author{
Evanthia Dimara, Harry Zhang, Melanie Tory, and Steven Franconeri
}

\begin{abstract}
When an organization chooses one course of action over alternatives, this task typically falls on a decision maker with relevant knowledge, experience, and understanding of context. Decision makers rely on data analysis, which is either delegated to analysts, or done on their own. Often the decision maker combines data, likely uncertain or incomplete, with non-formalized knowledge within a multi-objective problem space, weighing the recommendations of analysts within broader contexts and goals. As most past research in visual analytics has focused on understanding the needs and challenges of data analysts, less is known about the tasks and challenges of organizational decision makers, and how visualization support tools might help. Here we characterize the decision maker as a domain expert, review relevant literature in management theories, and report the results of an empirical survey and interviews with people who make organizational decisions. We identify challenges and opportunities for novel visualization tools, including trade-off overviews, scenario-based analysis, interrogation tools, flexible data input and collaboration support. Our findings stress the need to expand visualization design beyond data analysis into tools for information management.
\end{abstract}

Index Terms—Decision making, visualization, interview, survey, organizations, management, business intelligence.

\section{INTRODUCTION}

$\mathrm{V}$ ISUALIZATION varies in its goals, from testing data veracity or confirming a suspected pattern, to openended exploration in search of insight or enjoyment. Within organizations, often these processes serve an end goal of making a decision that will affect the organization's structure, processes, or outcomes. For example, a homeless shelter might need to decide which services might provide maximum benefit for an individual, while balancing resource distribution among many people. A university administrator might need to compare retirement plan offerings for faculty and staff, while juggling an overwhelming list of costs and benefits to many parties.

We argue that this decision making step has received too little attention in the visualization research literature. Across a survey and interviews of organizational decision makers, we identify challenges and opportunities for tools that can better support them.

We summarize the challenges with an example abstracted from our interviews. Sam is the CEO of a city convention center, facing the decision of whether to make a large investment in a greener power plant. Her decision is complex. Some important factors are quantifiable after hiring outside experts, such as a consultant who can estimate the tradeoff between initial capital costs against later savings from higher efficiency and government tax incentives, or engineers who can estimate the greener system's slower correction in interior temperature in response to rapid weather changes. But even these quantities carry uncertainties, or rely on sparse or unreliable data. Will that government tax incentive still exist after the next election? How much will the slower correction time upset our temporarily chilly

- E. Dimara is with Utrecht University and University of Konstanz. E-mail: evanthia.dimara@gmail.com

- H. Zhang and S. Franconeri are with Northwestern University

- M. Tory is with Tableau Software. patrons, or temperature-sensitive catering operations? Other factors are difficult or impossible to quantify. How does she weigh financial factors against an improvement in the organization's reputation, or the abstract moral goal of decreasing negative environmental impacts? Sam does not fully trust the recommendations of the analysts because of this lack of context: To account for government tax incentive uncertainties, she dove into the financial data analysis herself, but gave up after wading through the consultant's dozen disconnected spreadsheets.

The goal of the present study is to identify how visualizations can be embedded within the complex framework of organizational decision making (hereafter referred to simply as decision making). We identify themes and challenges, as well as opportunities for novel visualization tools to aid decision makers like Sam. These opportunities stress the need to expand visualization design beyond data analysis into tools for information management, including tools that facilitate trade-off overviews, scenario-based analysis, interrogation, more flexible data input, and collaborative work.

\section{Related Work}

We focus on decisions that influence the interpersonal, collaborative structures and processes of an organization (not just micro-decisions related to the decision-maker's own personal workflow) and aim to characterize organizational decision makers as visualization users. To that end, we discuss works at the intersection of visualization and decision making and then literature investigating the use of visualizations within organizations.

\subsection{Visualization \& Decision making}

Scholarly books on visualization emphasize that decision making is the ultimate goal of data visualization [1], [2], [3], [4], [5], |6], while the effective support of those decisions has 


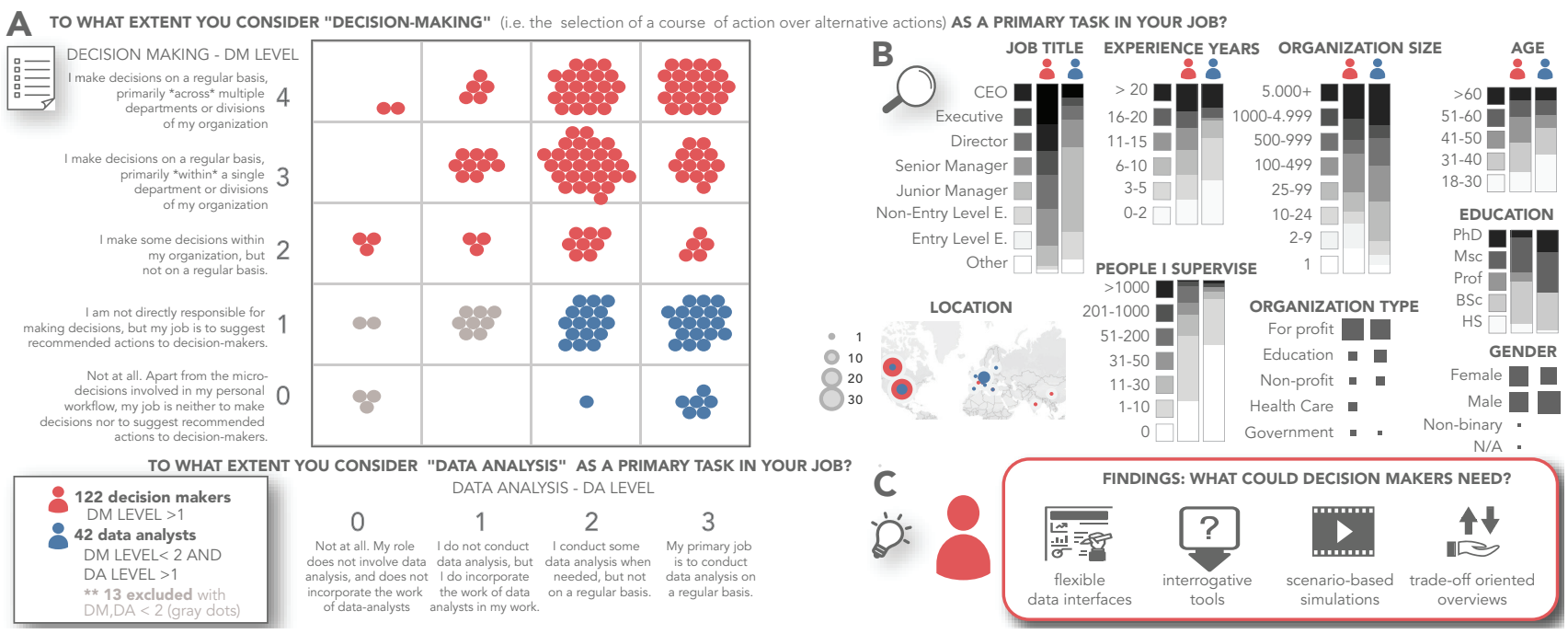

Fig. 1: A) Participants grouped as "decision makers" or "data analysts" based on their answers to the Decision Making (DM) and Data Analysis (DA) questions. B) Survey demographics. C) Emerging themes from our interview analysis.

been identified as the core challenge of visual analytics [7]. Decision-making is studied in domains such as psychology, economics, cognitive science and management, and each discipline has its own understanding of decision-making processes and how to study them. Yet visualization research emphasizes building a unified cross-domain understanding of human decisions made with visualized data [8].

Numerous visualization tools can potentially support decision making activities. General-purpose tools typically support any multi-attribute choice task [9] through displays such as decision trees [10], interactive querying [11] or more targeted solutions that allow users to express attribute importance and visually combine attributes into aggregated scores [12], [13], [14]. More sophisticated solutions mitigate decision biases through algorithmic support; for example, to assist a credit analyst to rank qualified customers for a loan without discriminating against female customers [15]. Domain-specific visualizations are tailored to decisionmaking in applications such as epidemiological research [16], finance [17] or urban planning [18].

While these systems likely contribute to data-informed decisions, they focus on supporting data analysis steps rather than decision mechanics. Most domain-specific designs are based on iterative development with data analyst users. However, data analysts report that senior decision makers often ignore their analysis unless it is oversimplified [19]. Most evaluations rely solely on visual analytic tasks (e.g., identify a correlation) rather than decision tasks [9]. Yet, empirical research has shown evidence that users who successfully complete visual analytic tasks can still fail on an almost identical task that is framed as a decision [20].

A reason why visualization research lacks an explicit tie to decision making could be the lack of relevant foundations in visualization literature. Although uncertainty research suggests that decision frameworks are essential for achieving realism and control [21], the visualization literature provides very few comprehensive frameworks that help us understand how humans make decisions over visualized data [8], [22], [23] and even fewer evaluation methodologies and metrics to assess their effectiveness [9], [24].
Notably, the few studies that do assess decisions concern cases of narrow complexity [25], such as binary decision tasks [23], [26]. Here we attempt to understand more complex forms of decision making [27] by studying its operational perspective within organizations.

\subsection{Visualization \& Organizational Context}

Numerous studies have investigated the use of visualization within organizational contexts. Most surveyed or interviewed professional data analysts about their data analysis workflow and problems [19], [28], [29], [30], [31], often targeting specific analysis challenges such as the role of exploratory analysis [32], [33], provenance [34], uncertainty [35], or big data |36|. Other works analyzed artifacts circulated within organizations, including visualizations [37], dashboards [38], or analyses of the features of commercial visualization software [30]. This corpus of "in the wild" observations have identified critical challenges for visual analytic tools, including demands to support statistical rigor (e.g., formal hypothesis testing, confidence intervals, normalization baselines) [29], [30], [36], data collection rigor (e.g., data cleaning and shaping) [29], [32], provenance [19], [32], [36], uncertainty exploitation [35], and audiences other than professional analysts, such as dashboard users [38].

Common to all these studies is their choice of target user: the data analyst. Data analysts are typically characterized as people whose primary job function is to answer questions with data [29], [32], [35], [36]. End products of their analysis may (or may not) support decision makers [35]. Unfortunately, this focus on analysts means that decision makers are currently understood incidentally and through the lens of their subordinate data analysts. Analysts have described decision makers as uncertainty-averse people [35] who do not use visualizations unless they display oversimplified information and gross trends [19], [37], noting that the cultures of analysis and decision making might not always agree on a visual language [37|. 


\section{Organizational Decision Making}

To understand decision making with data within a complex organizational context, we briefly review critical ideas from the field of organizational theory.

\subsection{Roles and Rules in Organizations}

Organizations are social systems established to make decisions. Modern organizational research recognizes that complex decision making can easily overwhelm the capacity of any individual [39], [40]. Organization theory draws on sociology, economics, political science and psychology to profile decision making processes. The field tends to focus on the social interactive and structural factors that affect collaborative decisions and actions rather than cognitive and psychological factors (e.g., [8]).

Because complex multi-person decision making faces the danger of information loss, miscommunication, uncertainty, and friction [41], [42], [43], organizations delineate specialized roles, rules and communication channels to enable these complex collaborations. They create horizontal and hierarchical divisions of labor: horizontally, subunits search for and process information based on specialized roles (e.g., marketing, design, engineering, or human resources). Hierarchically, decision makers at higher levels rely on synthesized and simplified information from the subunits.

\subsection{Organizational Decision Makers' Skills and Tasks}

Many organizational decision makers have non-technical backgrounds [44]. Even the quantitatively experienced are likely to be unfamiliar with the technical details at other specialized subunits. Therefore, they typically rely on aggregate information and metrics provided by subunits.

Organizational decision making differs from technical tasks in that the problems usually have greater complexity and ambiguity. As a result, higher-level decision makers frequently rest on "intuition" [45] rather than "definitive objective criteria" [46], and are highly exposed to sociopolitical factors [47]. Internal and external politics among different stakeholders will always orient the attention of decision makers and their combinatorial use of different types of information [48].

Several canonical models depict how socio-political factors influence the use of information in organizational decisions. A 'political model' focuses on conflicting interests and power variation [49], which demand that higher-level decision makers engage in a form of conflict resolution [50]. A 'programs and programming' model [51] focuses on how organizations standardize routines and institutionalize rules to reduce the friction and redundancy over different tasks. The 'isomorphism' perspective stresses external influences [52], [53], where instead of searching for information and calculating the benefit and cost of alternatives, they follow actions taken by other organizations. The 'Garbage Can' model [54] denies the view that decision making is predictable or structured by highlighting the stochastic processes in which idiosyncratic information happens to become salient and useful at the time of decision making.

These models of the decision making mechanisms in organizations profile very different tasks and questions than the typical quantitative optimization problems during technical decisions. They often ask: who are the stakeholders involved in this problem? How influential are they? How important are their demands? What are their individual goals and how do those align with the organizational goal? Have they communicated with other parts of the organization? What are the unsolved problems and unused solutions we currently have? What are our competitors' decisions?

As the roles, tasks and processes greatly differ between decision makers and data analysts, the visualization tool needs of decisions makers likely differ drastically from the features that help data analysts with data processing, analysis and presentation. This leads to our research question: Who are the organizational decision makers and what are their data visualization needs? We seek answers to this question empirically using a survey (Section 4 ) and interviews (Sections 5 and 6 .

\section{ASKING THE EXPERTS: SURVEY}

We surveyed real world decision makers to understand their work practices. All materials and data are available here: https://osf.io/nqtj6/?view_only= da1856fc1b15489b96fb82657891b51c Please refer to the survey instrument at this link to see the exact questions asked. Results are shown in Fig[1 (A, B) and Fig 2 (A, C).

\subsection{Survey Design}

We obtained IRB approval to survey decision makers and data analysts on their tasks, needs and visualization usage. While our focus is on decision makers, we included analysts in order to examine organizational decision making practices from their perspective. We advertised the survey via LinkedIn, Twitter, Reddit, and the commercial research platform userzoom.com via the following call "It's tough to organize data to make complex decisions in your organization. We want to design a software tool to help. Help us customize it to your needs by completing a 15'survey at $X$ and please forward to your manager $\&$ colleagues", encouraging readers to forward the link (snowball sampling).

We first explained the purpose of the survey was to better understand how software tools are used in professional settings. Participants reported job title, rank, number of people they supervise, years of experience, and the organization size and sector (for-profit, government, etc.), as such organizational characteristics may significantly affect the task and roles of employees in acquiring and using information [55], [56], as well as standard demographic information (Fig 13 ).

We classified users based on their responses on how their work relates to decision making and data analysis roles (Fig[1A). They indicated the extent to which they considered 'decision-making' (i.e. the selection of a course of action over alternative actions) as a primary task in their job. Those who indicated some decision making role (read DM levels 2-4 in Fig 1 A) in their work were classified as "decisionmakers". Respondents then indicated the extent to which they consider 'data analysis' as a primary task in their job. Those who described their role as not responsible for making decisions while pursuing a data analysis role (read 
DA levels 2, 3 in Fig [1A) were classified as "data-analysts". Based on their roles, to verify quality of responses, we asked for explicit examples of decisions and data analysis.

Participants reported in open-ended formats which tools they use for certain tasks ( $\mathrm{Fig} \sqrt{2} \mathrm{C})$, decision making and data analysis (conditionally base on their role), as well as data communication and data visualization (all participants). They also responded in Likert scales how often they use spreadsheets as well as various visualizations including dashboards, interactive vs static visualizations, visualization software, and 20 types of basic charts (Fig 2A). Finally, they were asked to suggest potential improvements for new tools and indicated their interest in a follow-up interview.

\subsection{Results}

We received 177 complete responses, after removing 30 participants whose responses were incomplete or nonsensical. Their profiles are summarized in Fig 1 A consisting of 122 decision makers (red dots) and 42 data analysts (blue dots). 13 respondents did not meet the inclusion criteria of either group and were excluded (gray dots). Our sample held diverse roles and responsibilities, and worked for organizations of different sizes ( 1 person to $>5000$ ), locations and domains (e.g., commercial, nonprofit, health, education) (Fig 1B). In (Fig 1 A), we observe that the "decision makers", as derived by our classification rule, had different professional and demographic profiles than the data analysts.

We expected that decision making and data analysis roles would also be fairly distinct within the organization, and that decision makers would mostly rely on others to do data analysis on their behalf. We were surprised to see that many decision makers reported conducting data analysis themselves (see top-right red dots in Fig/1A). Some of this analysis work may still build on substantial work by others, as we did not ask directly about how other people might pre-process their data and artifacts. It is also possible that our call has attracted decision makers with more interest in data and analysis than average. Conversely, people working in analyst and data scientist teams reported being involved in decisions that go beyond micro-decisions involved in their personal workflow (and are therefore "decision makers" by our classification, along with the decision makers who hold less data-oriented roles). That suggests that aiding decision support could also benefit the data analysts' workflow. P159 noted on a common misconception about role diversity, "Most discussions of decision making assume that only senior executives make decisions or that only senior executives' decisions matter. This is a dangerous mistake.".

Fig $2 \mathrm{~A} \& \mathrm{C}$ illustrate the differences between decision makers and analysts in their use of visualizations. Fig $2 \mathrm{C}$ reports qualitative analysis of open text responses of number of tools per user profile. Fig 2A, for each mean frequency of usage, a point estimate is reported together with a $95 \%$ confidence interval (CI), indicating the range of plausible values for the population mean. We observe that decision makers showed preferences for different types of charts. For instance, analysts tend to use histograms, stacked bars and scatterplots, while decision makers tend to use more flow charts and pie charts. This could reflect a difference in tasks, literacy or even interest in different type of represented information, with data analysis seeking to understand data distributions and patterns (e.g. a correlation in a scatterplot), while decision makers emphasizing future planning and proportion summaries. Certain chart types were frequent or rare for everyone within the organization (e.g., high usage of bars and lines and rare usage of treemaps, radial charts and parallel coordinates). Yet, the types of visualizations being used (Fig 2A) can also be influenced by the defaults of the available software (Fig 2 C.) Two points that stood out were the high use of spreadsheets among decision makers and the considerably lesser use of visualization software and interaction by decision makers as compared to analysts (Fig 2A). The use of spreadsheets some data visualization software suggests an interest within decision makers in data, but that they use less sophisticated tools. The limited use of interactivity could further suggest that the established organizational practices for decision making are centered around static data reports.

Fig $2 \mathrm{C}$ shows an aggregated analysis of 940 reported tools in total that assist with tasks: decision making, data analysis, data communication, and visualization . Yet 9 participants expressed frustration for switching among various tools to support different phases of their workflow. To our surprise, we found no "decision making" tool designed specifically to support that activity. Rather, various general purpose tools contributed to decision making tasks, mostly non-data analysis tools along with spreadsheets (Fig $2 \mathrm{C} 1$ ). By further looking at the design focus of the non-data analysis tools used by decision makers (Fig 2 C1 top-right), we might infer some essential needs such as the support of natural language, drawing, collaboration, and project management. The widespread adoption of these tools suggests a need for potential decision making support tools to integrate or interoperate effectively with these software ecosystems.

We further used open card-sorting analysis [59| to generate categories of suggestions of critical challenges within organizations that need to be supported. Percentages indicate participants who made the same suggestions. Consistent with previous work [19], [28], [32], |36], [37], data analysts emphasized the pain of data preparation and cleaning, integration from disparate sources, and the need to visualize effectively missing, uncertain, or low quality data (38\%). Decision makers also confirmed that data "wrangling" steps are burdensome of data-driven decision making $(23 \%)$ which should be also addressed at an organization level. As noted by P101, "Good visualizations don't fix problems with underlying data. Organizations need to invest more in data governance and data quality efforts.". Some data analysts $(10 \%)$ voluntarily requested guidance on how to visualize effectively, while many decision makers $(21 \%)$ complained that experience difficulty to understand data analysis results. As noted by P126, "Even when the output is of high quality, leaders mostly just decide on what they see and do not undertake a statistical analysis, partly because they do not fully understand themselves and partly because there are no statisticians at hand to support timely decision making".

Unlike data analysts, decision makers further noted that their disparate data sources include qualitative data, requesting their integration with results by data analysts within a single interface. P267 noted for qualitative data creation: "Data input via mobile devices would be a huge benefit, and that data would flow into simple cloud-stored tables (e.g., 


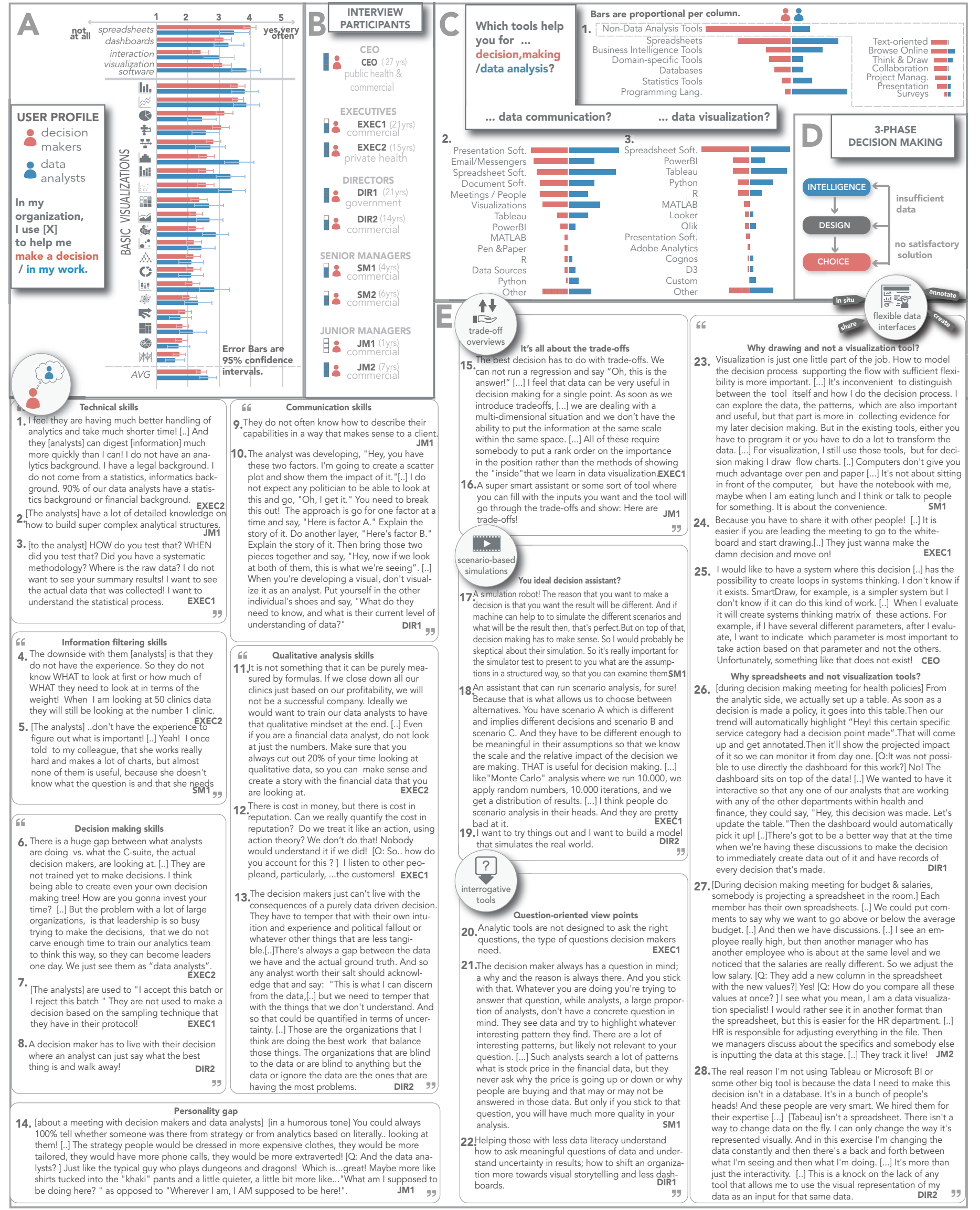

Fig. 2: A Survey: frequency of spreadsheets, dashboards, interaction, visualization software and basic charts usage. B Interviewee nicknames, roles, experience, relation with data analysis (0-3 blue boxes). C Survey: 940 reported tools (in total) that help participants with tasks: decision making and data visualization (1) data communication (2) and data visualization (3). D 3-phase decision model [57], [58]: our decision makers occasionally use data visualization for some intelligence tasks, but almost never during the later phases. E Interviewee quotes on: (1-14) the perspective of decision makers towards data analysts and (15-28) themes outlining opportunities for novel visualization tools. 
spreadsheet) that could easily be accessed and analyzed. Because a lot of my data-gathering is via conversation and observation, being able to seamlessly enter the data as it's being observed or expressed would be a great time-saver. But not into proprietary or needlessly complex tools. Simple data, thoughtfully gathered, often results in the most profound and actionable analysis.".

Numerous other decision makers $(41 \%)$ stressed such need of enriching interactivity of current tools. Along with the need to create data on the spot discussed by P267, they requested a seamless visual environment that will allow drag and drop operations and analysis annotations. P129 asked for a tool "to help me tell my data specific story. Have it be something that flows from the base application right into a tool like PowerPoint or Word and have it create visuals that utilize the best practices such as chunking, use of white space...ability to add highly customized story-titles, and annotations...have it tell the story and be able to show elements of the story in animation...to tell the story piece by piece.". This also echoes with the more political and ambiguous nature of decision makers' jobs. While "telling stories" is also important during some data analysts' work [28], [32], decision makers are the ones responsible for skillfully framing their proposals during the decision making meeting where they have to synthesise the interests and perspectives from different stakeholders [60], [61]. The majority of requests on enriching interactivity requested an interface that will allow them to organize their data freely $(16 \%)$. This creates the need for tools that have better flexibility in switching and combining different sources and types of information.

Our most critical finding is the lack of a data interface with a design focus on the decision making process. To be able to inform the creation of such a tool, there were questions that remained unanswered about the needs of decision makers within data visualization. On one hand, decision makers showed a strong interest in low-level data work (e.g., spreadsheets, active involvement in data analysis efforts). On the other hand, they tend to use less sophisticated tools and static simplified displays. Still, they request highly interactive features when working with data. One explanation can be that decision makers are simply not aware of more interactive solutions, and find themselves most familiar with spreadsheet tools. Yet it remains unclear if there are other limitations of visualization software that hinder its adoption for decision making support.

\section{Asking the EXPERTS: INTERVIEW DESIGN}

Our survey investigated the current use of visualization in the landscape of organizational decision making. Our next step was to conduct an interview with some of our survey participants to understand decision maker profiles in more depth, as well as to identify challenges and opportunities for novel visualization tools. Here we present the interview approach, and the next section will present our findings.

\subsection{Interview Participants}

From the pool of the survey's decision makers (Fig 13 ), we obtained IRB approval to reach out to those who agreed to be contacted for an interview. 10 answered our email and participated in an interview. As it was not our intention to interview data analysts, we discarded one participant who had accidentally self-identified as a decision maker, but then clarified that they only conduct data analyses for decision makers. While we cannot assess how representative our interview volunteers were compared to the broader sample of survey respondents, the interview sample was diverse (Fig $2 \mathrm{~B}$ ) holding various management positions including CEO, Executives, Directors, Senior and Junior Managers, located in USA, Canada and Europe, of ages 32 - 66. At the time of the interview, three participants were working on critical decisions in the context of the COVID-19 pandemic (CEO, EXEC2, DIR). The organization sizes ranged from 25 to 5000+. Most organizations were commercial, two in healthcare (public and private) and one in government.

\subsection{Interview Questions \& Analysis}

We first explained the purpose of the study as to help both academic and industry researchers, as well as designers, understand the software needs of decision makers. We summarised what we learned from their survey, as a way to connect with the participant, save introduction time, and confirm that survey responses still reflect their current status. We introduced the interview's purpose as "software designers who want to understand how to support decision makers". We intentionally did not mention data visualization in the introduction. There were 4 core questions:

Q1: We used the critical incident interview technique [62] asking them to narrate a difficult decision they made in the past. Depending on the examples, we asked clarifications on how they navigated that decision (e.g., tools and/or information they needed). Responses often generalized decision making practices beyond the specific example.

Q2: To better understand the mental model [63] of the decision maker, we asked them to draw the way they mentally organized the information involved in the decision example in Q1. Two participants lacked a pen so they described verbally what they would draw.

Q3: We asked them to identify differences between decision makers and data analysts, as they either had worked with a team of data analysts or had been data analysts themselves before becoming decision makers. Two participants who did none of the two were not asked this question.

Q4: We asked them to describe their ideal decision making assistant. We clarified that there is no need to restrict themselves to the capabilities of current technology. It could be a future technology, an artificial intelligence, or a person, and we encouraged them to let their imagination run free and request what would be most helpful.

Interviews, conducted by skype, lasted about 1 hour. With participant permission, interviews were audio recorded and transcribed. The transcripts were annotated independently by all 4 authors ( 3 visualization and 1 management researchers). One visualization coder used Thematic Analysis |64| to analyze the transcripts, identifying meaningful patterns (themes) from the data. In their first independent pass, the other 2 visualization authors identified their own interesting themes and events. The independent observations were either integrated into existing themes or resulted in new themes. All 3 visualization researchers took an inductive and iterative approach to derive themes purely 
from the data. The management researcher took a mixed inductive and deductive approach $|64|$ to identify some themes through the lens of management theory.

\section{ASKING THE EXPERTS: INTERVIEW FINDINGS}

This section presents our interview results on who are the expert decision makers and their data visualization needs.

\subsection{Decision Makers within Organizations}

We first provide an overview of the decision maker profiles and the way they make decisions within their organization. Later sections focus on how such decisions can be better supported by visualization.

\subsubsection{Who are they?}

Prior to our study, we expected decision makers to come from management schools and have a limited understanding of the technical aspects of data analysis. To our surprise, while our participants had diverse backgrounds besides management (e.g., CS, law, economics, aerospace engineering), throughout the interview they appeared quite knowledgeable in data analytics and visualization. All of them spontaneously expressed the need to involve more data and visual analysis in their decision process, and even the need for guidance to conduct rigorous data analysis themselves. Note that our sample consists of volunteers who answered our call, so this sample might not be representative of all decision makers within organizations, but it is likely reflective of our target users: people who want to make more data-informed decisions, but for whom the current technology falls short of supporting them effectively.

Another characteristic of our decision makers was the importance of time in their daily work (see also survey quote P126). That was evident even from the way they managed the interview, making sure to finish in 1 hour precisely. Interestingly, they characterized their tasks by the time they chose to allocate on them, rather than their inherent task difficulty or effectiveness (Fig22E.6). EXEC2 drew a decision tree of her actions indicating percentages of time allocation (e.g., allocates $50 \%$ of her time on action X). Their workflow appeared to be driven by well defined questions which they were always able to articulate as being relevant to concrete actions. Questions and actions were the two criteria for their engagement (or not) with the findings of the data analysis teams. Does this data visualization help me answer my question? Does this analysis help me identify my next actions? (Fig 2E.21). We discuss the need for visualization to support question-driven analysis in Section 6.3.2.

Interviewees had roles at various levels of the organizational hierarchy, from junior managers to CEOs (Fig[2D). Although the interview always started with the same question (Q1), their role seemed to affect the scope of the interview. Upper management focused more on high-level observations (i.e. discussions on national policies, systems, general assessments) and we had to make an effort to extract their concrete tasks and tools. In contrast, with lower level management their narration started directly with concrete tasks and we had to ask explicitly to generalize their observations. These top-down vs bottom-up perspectives might influence the type of visualization those users need.

\subsubsection{How do they make decisions?}

Across all participants, we consistently observed that no decision was made by a single person alone. EXEC1, to make a decision on their manufacturing strategy, must combine the perspectives of the production, marketing and logistics teams. EXEC2, to make a decision on the sustainability of their thousand private clinics, needs to combine the perspectives of the operations team, the clinical analysis team, as well as the real estate team that analyzes their long term investment potential. Then each of the 9 strategists makes a recommendation for their respective locations. Their aggregated plan passes to the C-Suite team for refinement and finally to the CEO who gives the final "pass or fail". Such group decision making activities are very much iterative, involving several meetings and back and forth communication. Decision makers often felt that the amount of iteration in such meetings is counterproductive. For example, JM2 wanted a decision assistant that would reduce iterations, so "we move on to something else, instead of doing three meetings about the same thing!"

Moreover, while they sometimes circulate information artifacts in advance of the meetings (typically a report), the decision process per se (i.e. synthesis, evaluation of alternatives, and conflict resolution) happens in a rather $a d$ hoc way. By the end of a meeting, the decision must be made. Such "garbage can" processes [54] seem, at least in part, to be the result of the absence of a sophisticated tool that can organize and present complex data and analyses for the decision makers. Indeed, the interviews reveal that although most decisions involved complex data and analyses, the group decision process was supported only by discussions or spreadsheet software. Data analysis results were typically circulated before the meeting as static reports. Participants also used personal paper notes or whiteboards. Only one participant reported the use of dashboards during the decision making meeting. However, the use of visualization was primarily for data communication or to justify decisions to their peers in a post-hoc way, rather than during the actual decision process. We elaborate more on the need to support collaborative visual data analysis in Section 6.3.1

Just as in group decisions, in individual decision making processes, visualization and technology in general did not seem to play a major role. Consistent with the survey findings, our interviewees were not supported by any "decision making" tool designed specifically for that task. Data visualization was sometimes involved at early stages of the decision process. For example, sM1 reported using visualizations only when "collecting evidence" for his "later decision making" (Fig2E.23). Decision making involves other data-heavy tasks besides collecting information. One wellaccepted account suggests that humans go through three essential phases in the act of decision making: INTELLIGENCE, DESIGN, and CHOICE [57], [58] (Fig[2D). We were able to identify those phases in all decision examples narrated by the participants. Visualization was sometimes used to understand some data prior to the decision (INTELLIGENCE phase), but not to support the synthesis of alternative solutions (DESIGN phase), or their final selection (CHOICE phase). We elaborate more on the need for visualization tools to support the phases of DESIGN and CHOICE in 
Sections 6.3 .3 and 6.3 .4 respectively.

Decision makers expressed the need "to model the decision process supporting [their] flow" (Fig[2E.23). Developing a single system that would be able to support the user in all phases of the decision making would be valuable. However, decision making is a creative process, unique to each individual, and it is unclear how a visualization tool can become a useful assistant throughout the decision process. The following sections attempt to outline this landscape by identifying challenges in data analytic practices (Section 6.2 as well as our main themes that highlight opportunities for design innovations (Section 6.3).

\subsection{Decision Makers vs. Data Analysts}

We explored how decision makers describe their relationship with their data analysts. The benefit from understanding this relationship is twofold. First, it complements previous interview studies that investigated visual analytic practices from the perspective of data analysts [19], [35], [37|. Second, we hope that by capturing decision makers' concerns about their data analyst teams, we can uncover shortcomings of analytic practices that prevent effective decision support. Fig $2 \mathrm{E}$ shows a subset of participants' quotes. All participants expressed (spontaneously) the need to base their decisions on reliable data and analysis. JM1's company went one step further, making a significant investment and collaborating with visualization researchers from academic institutions to train its leaders around analytic thinking. The course focused on explaining basic analytic concepts and how leaders can communicate with their analytics team in a strategic way. JM1 humorously noted that the moment she entered the room of the course she could immediately tell who was a decision maker and who was a data analyst by their looks, as decision makers had much more extroverted personalities (Fig2E14). The themes that will be described next identify challenges in current practices (i.e. in the way people do data work), while the next section will focus more on visualization design opportunities (i.e. the visualization tools people use to do data work).

NEED FOR EXPLANATION OF ANALYSES : Interestingly, the second reason for this course was that JM1's company wanted to exploit the communication skills of its decision makers to explain the visual analytic solutions of the company to broader audiences (Fig22E9). Consistent with the survey, the way that data analysts present their results can be confusing for decision makers and stakeholders with diverse backgrounds (Fig 2E1,2). DIR1 used to advise his data analysts not to "visualize as an analyst!" For example, in policy decision making, a relationship presented in a scatterplot might not be immediately understood by a politician. So DIR1 advised the analyst to break the analysis out, explaining each factor separately and narrating the analysis conclusions step by step (Fig 2 E10). This type of explanation of an analysis can also be demanded by decision makers with expertise in analytics or statistics, who do not trust summary results without an unpacking of the steps followed to derive the conclusions (Fig 2E3). Although the level of explanation that each decision maker needs can differ, all participants described a process where explanations occur "on-demand" and are targeted to the question at hand. More technical and elaborated explanations (e.g. analytic provenance) could instead violate the need for less verbose analysis that we discuss next.

NEED FOR INFORMATION REDUCTION: Management research has noted information overload as a perennial issue for decision makers at different levels of organizations [65]. In our interviews, decision makers similarly noted that the visualizations presented by their data analysts were sometimes too verbose. They often conducted analyses that seemed unfocused or unnecessarily detailed (Fig2E4), or presented too many charts that they deemed irrelevant to the main question (Fig2 2 E5). The impression of some data analysts that decision makers "ignore" their analysis [19], [37] might also be due to a lack of relevance and context of that analysis, as JM1 has emphasized: "to present WHAT IS IMPORTANT to make decisions and not just everything!". Information reduction further echoes with decision timeliness discussed in Section 6.1.1

NEED FOR QUALITATIVE ANALYSIS: Decision makers also reported a lack of qualitative nuance (Fig 2E11,12,13), which resonates with the "Garbage Can Model" where important information can be difficult to track, analyze and distribute in the most rational or structured ways for the organization. EXEC1 explained that some factors can not be purely quantified (e.g., the company's reputation) and that he incorporates the client's informal feedback into that analysis. Similarly, EXEC2 noted: "This is patients! Each patient is very different... although we have our ratio... saying we need one nurse per 4 patients, one patient can take one nurse because they have more needs or they can't walk. There are a lot of different stories and I need to make sure that I do not look purely at the numbers.". In contrast, analysts in previous research describe decision makers as being generally uninterested in data, and unwilling to use visualization tools, until they need to justify decisions they have already made [35], [37].

Of course, the reality likely lies in between these perspectives. We encourage future studies to draw more insights from the management literature in considering how visualizations can adopt certain designs to reduce such poor practices. For example, research shows that open, cross-level communication (instead of hierarchical channels that strictly follow the organizational chart) helps organizations make better decisions [48], [66], [67]. Tool designs that account for managerial incentives and facilitate monitoring structure and composition of decision making teams can help reduce the misuse of power and information by decision makers [68|. This implies that transparent data analysis through visualization can encourage different people to monitor and attenuate the misuse of information. We note that the use of information relates to the organizational culture, and in particular, the level of misuse of information to serve personal and political interests within organizations [69], |70|. Such insights indicate substantial opportunities for visualization designers to help decision makers engage in more effective and efficient use of information.

\subsection{Future Visualization Tools}

Both survey and interviews suggested that decision makers lack "decision making" tools designed to support the flow along all decision phases: INTELLIGENCE, DESIGN and 


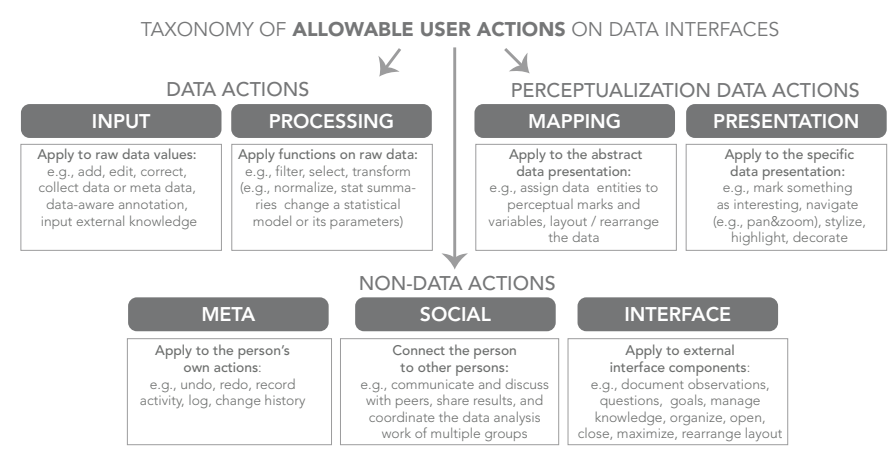

Fig. 3: Taxonomy of allowable user actions in a visualization system [71]. Increasing the cardinality of such actions increases the degree of flexibility of the system. Input and social actions appeared as crucial for decision makers.

CHOICE (Fig.2D). We next present emergent themes derived from our data that highlight design opportunities for visualization to better support decision makers.

\subsubsection{Flexible Data Interfaces}

Our interview provided a potential explanation for the survey's contradicting findings that while decision makers request interactivity, yet they favor static solutions over sophisticated visualization software: Current visualization tools might lack the necessary "flexibility" to support decisions "flow" (Fig. 2E.23). To understand how to increase visualization flexibility to accommodate the creative act of decision making, we can draw upon the concept of flexibility for data interfaces that we have proposed in previous work |71]. It was developed as a taxonomy of interactions with visualization systems, so this schema was used to help us categorize and identify the most prominent of those interactions for decision makers. Interactions are classified into INPUT, PROCESSING, MAPPING, PRESENTATION, META, SOCIAL and INTERFACE actions (see Fig. 3). One way to increase the flexibility of a visualization system is to increase the cardinality of allowable actions [71]. We report next the action categories that have been identified by our participants as critical for their decision making process in an order of importance (declining frequency of incidents).

SOCIAL ACTIONS: Interactions that connect the user to other users (Fig. 3) were suggested as vital in decision making workflow (Fig.2E.24). Section 6.1.2 showed decisions within organizations to require a group of decision makers to iterate together. To share thoughts and communicate proposals, decision makers often rely on the expressiveness of paper drawings and whiteboards (Fig. 2E.23,24,25), but these media are not connected to data sources. Visualizations were used in some meetings to present results of data analyses, but pre-defined visualizations lack the adaptability afforded by a whiteboard. EXEC1 mentioned that his peers do not always trust summary results of other teams, but they do trust his recommended actions if he illustrates them step by step on a whiteboard. That is at least partially because group decision making is not only about reporting each team's analysis findings independently - our participants expressed the need for coordination in data analysis. As mentioned by EXEC1: "The best use case for us is to understand the data together. The same data! Rather than: 'I have my data and somebody else has their own data."' To that end, visualization research on collaborative data analysis (e.g., [72]) could be essential to decision making support. This does not necessarily refer to a synchronous co-located collaboration, but that coordinated analysis is needed that shares a common ground dataset.

INPUT DATA ACTIONS: Interactions that operate on raw data (see Fig 3), often overlooked by visualization tools, range from correcting erroneous data, to adding data-aware annotations to creating knowledge from scratch. We illustrate the value of input actions with three examples. After our survey, we anticipated that the preference for spreadsheet software by decision makers in the survey was mainly due to familiarity biases. However, our interviewees explained that spreadsheets are more flexible in supporting essential data operations. In three different meetings, budgetary decisions (JM2), public health policy making (DIR1) and strategic product design (DIR2), spreadsheets were projected to the decision makers. While alternative actions were being discussed, they were correcting, annotating and creating data rows and columns in the spreadsheet (Fig 2E.26,27,28). DIR1 explained that the preference for spreadsheets was more of a necessity rather than a preference. They had displayed interactive dashboards at early stages of the decision process (i.e. INTELLIGENCE phase) in the same meeting. In fact, while they came up with a novel technical solution of connecting the dashboard with the spreadsheet software, they would prefer if the input action was performed directly on the visualization. To that end, JM2 explained that in order to maintain the flow of the managers' discussion, HR assistants added data to the spreadsheet, tracking the decision in real time. In DIR2's case, the manager collected data on employers' opinions and decision criteria in a spreadsheet for a period of 2 years. Then, all teams iterated over the spreadsheet to synthesize and resolve conflicts. Data creation also appeared essential for qualitative analysis (Section 6.2), as decision makers often transformed qualitative information into quantitative formats; e.g. both CEO and EXEC2 extracted "numbers" from text reports or assigned weights of importance. From a broader perspective, INPUT actions allow decision makers to refine and integrate their knowledge into the visualization system. These results highlight a gap in current visualization software, which makes an assumption that data already exist and are structured, all before analysis takes place. In contrast, we found decision makers needing to write back to the data source or to restructure it on the fly as part of the decision making process.

MAPPING \& PRESENTATION ACTIONS: These interactions apply to the representation of the data (Fig 3). When we asked decision makers to draw the way they organize information in their head, we expected various shapes that we would later translate into novel visualizations. To our surprise, the mental models of our decision makers were quite similar. We saw flow charts, a bullet list and a decision tree (a flowchart-like diagram), action-oriented representations not typically supported by visualization tools, likely because they are not perceived as data-driven. Other action oriented domains, such as medical diagnosis, have embedded flow charts within the visualization [73]. The CEO, when describing his ideal decision assistant, said: "I would like to have a system where this decision [..] has the possibility to create loops 
in systems thinking. I don't know if it exists. SmartDraw, for example, is a simpler system but I don't know if it can do this kind of work. [..] When I evaluate it will create systems thinking matrix of these actions [..] Unfortunately, something like that does not exist!", indicating that free form sketch-based data visualization $\mid 74]$ could be helpful in decision support.

PROCESSING: Participants did not mention many processing actions, possibly because those are closer to the technical needs of data analysis. Yet EXEC2 described her ideal decision assistant as "..if tableau or excel could suggest to me, because I do not have a data analyst background, like most others in C-suite [..] Hey in order for you to look at these data, it's better to calculate the weighted average this way instead of putting an average of all clinics", uncovering the need for guidance on rigorous data processing actions.

Another way to increase the flexibility of a visualization system is to increase the cardinality of interaction means with which a user can perform each action [71]. SM mentioned that the reason he can not use visualizations is because it is inconvenient to interact in front of a desktop computer (Fig2E.23). Promising solutions can be suggested from research in situated visualizations [75]. We note here that the seemingly conflicting calls for information reduction and for increasing flexibility differ. Information reduction refers to the amount of data (in our case within visual representations), while flexibility refers to the ways with which a decision maker can interact with the displayed data.

We next discuss themes associated with each of the decision phases proposed by H. Simon, a precursor of decision making models about human and artificial intelligence: INTELLIGENCE, DESIGN and CHOICE. We note that while these ideal typical classes of activities in Simon's model conceptually help us disentangle the decision making process, they are certainly more tangled and iterative in real life.

\subsubsection{Interrogator Tools}

During the INTELLIGENCE decision phase, the decision maker works on collecting and understanding the data relevant to the decision (Fig 2P). Our participants used visual analytics during that phase but these tools did not always meet their needs. The problem could be understudied: either it lacked the qualitative dimensions (Fig 2E.11,12, 13) or it was too overwhelmed with verbose and irrelevant analysis (Fig 2E.4,5). These shortcomings relate to the main target of the decision maker: the well-identified question. As noted by SM1: "The decision maker always has a question in mind; $a$ why and the reason is always there. And you stick with that. Whatever you are doing you're trying to answer that question, while analysts [...] don't have a concrete question in mind. They see data and try to highlight whatever interesting pattern they find. There are a lot of interesting patterns, but likely not relevant to your question." Similarly, EXEC1 noted that "Analytic tools are not designed to ask the right questions, the type of questions decision makers need." Here our participants did not mean that the tool should pose the questions, but rather that the tools could encourage question oriented analysis. Visualization research has explored designs with a question driven focus, including natural language systems (e.g., [76]). These tools are a step in the right direction, but do not go as far as helping people define the questions to ask. In a similar fashion, decision makers and data analysts could collaborate in question driven systems, where the system encourages or even assists less verbose analytic practices and where support for their asymmetric collaborative practices are explicitly built in. Moreover, to enable decision makers define rather than solve questions, the tools should focus on helping users navigate through the ambiguity in the usefulness of relevance of different information for their tasks. Therefore, the tools should facilitate 1) users' discretionary approaches of storing, organizing and annotating quantitative and qualitative data and 2) convenient, iterative scan, alignment and recombination of different types of information.

\subsubsection{Scenario-based Simulations}

During the DESIGN decision phase (Fig/2P), the decision maker generates alternative solutions to the problem. Participants emphasized that the ideal decision assistant should help them synthesize decision alternatives (Fig2E.17-19). SM1 noted "A simulation robot! [...] if the machine can help to simulate the different scenarios and what will be the result then, that's perfect. But on top of that, decision making has to make sense. So I would probably be skeptical about their simulation. So it's really important for the simulator test to present to you what are the assumptions in a structured way, so that you can examine them." Similarly, EXEC1 noted "Ah.. it would be an assistant that can run scenario analysis, for sure! Because that is what allows us to choose between alternatives. You have scenario $A$ which is different and implies different decisions and scenario $B$ and scenario $C$. And they have to be different enough to be meaningful in their assumptions so that we know the scale and the relative impact of the decision we are making. THAT is useful for decision making. [...] more like "Monte Carlo" analysis where we run 10000, we apply random numbers, 10000 iterations, and we get a distribution of results. [...] I think people do scenario analysis in their heads. And they are pretty bad at it." Assistance in scenario generation can be enhanced with interactive visualization planning solutions [77].

\subsubsection{Trade-off Overviews}

During the CHOICE decision phase, the decision maker selects the 'best' solution from amongst the alternative solutions using some criteria (Fig[2D). Similar decision types have been described in the visualization literature as multiattribute choice tasks [9] and have been supported by several promising visualization tools [12], [13], [14| that help users to define the importance of decision criteria and visually combine multiple attributes into aggregated scores. Participants did not report using such solutions since they are usually customized tools for specific applications. Yet participants expressed difficulty in dealing with trade-offs. EXEC1 noted "The best decision has to do with trade-offs. We cannot run a regression and say 'Oh, this is the answer!' [...] I feel that data can be very useful in decision making for a single point. As soon as we introduce tradeoffs, [...] we are dealing with a multi-dimensional situation and we don't have the ability to put the information at the same scale within the same space. [...] All of these require somebody to put a rank order on the importance in the position rather than the methods of showing the 'inside' that we learn in data visualization." JM2 described the ideal decision assistant as one that could help them see the tradeoffs, likely with some computational support, "That could be a super smart assistant or some sort of tool where you can fill 
with the inputs you want and the tool will go through the tradeoffs and show: 'Here are trade-offs!"'. However, facilitating multi-attribute choice has been challenging for visualization research as those tools are difficult to evaluate [9] and expressing attribute weights in an intuitive manner remains a design challenge |14|. Notably, our decision makers expressed mostly difficulty in dealing with the sheer number of attributes rather than the number of alternatives, which were typically limited to $2-5$. However, it is unclear whether those limited option sets grew out of necessity due to the lack of tool assisting in handling larger choice sets.

\section{Conclusion}

While visualizations support analysis and the transfer of knowledge between analysts and decision makers, this work outlines a much larger space where visualizations can play a role. To understand how data visualizations can fit into the broad landscape of decision making support within organizations, we asked expert decision makers to describe the way they make decisions in complex environments as well as the role of technology in their workflow. Participants reported that there is no 'decision making' tool that supports them throughout their 3-phase decision process, uncovering an undersupported community whose needs are highly aligned with the goals of the visualization community.

Our findings stress the need for data visualization to support decisions via qualitative nuance and information reduction, along with concise on-demand explanations of the data analysis process. We further identified opportunities for novel visualization designs, including flexible data input and collaboration mechanisms, interrogation, scenariobased analysis, and aids for trade-off overview analysis. Yet, our findings, as in most qualitative studies, should not be interpreted as conclusive or cases for drawing causal inferences, as they are grounded in the data that we gathered. Future research is needed to confirm and extend these findings to a wider set of decision makers and scenarios.

\section{ACKNOWLEDGMENTS}

We thank J. Stasko, D. Keim and DBVIS team for their helpful feedback, and our participants for their time and insightful discussions. Work is funded by the European Union's Horizon 2020 research and innovation program under grant agreement No 825041.

\section{REFERENCES}

[1] K. A. Cook and J. J. Thomas, Illuminating the path: The research and development agenda for visual analytics, 2005.

[2] T. Munzner, Visualization Analysis and Design. A K Peters/CRC Press, 2014.

[3] M. O. Ward, G. Grinstein, and D. Keim, Interactive Data Visualization: Foundations, Techniques, and Applications. A. K. Peters, 2015.

[4] R. Spence, Information Visualization: An Introduction. Springer International Publishing, 2014.

[5] C. Ware, Inf. Vis. Elsevier, 2013.

[6] D. Rees and R. S. Laramee, "A Survey of Information Visualization Books," Comput Graph Forum, vol. 38, no. 1, pp. 610-646, feb 2019.

[7] D. Keim, F. Mansmann, J. Schneidewind, and H. Ziegler, "Challenges in Visual Data Analysis," in IEEE IV, 2006, pp. 9-16.

[8] L. M. Padilla, S. H. Creem-Regehr, M. Hegarty, and J. K. Stefanucci, "Decision making with visualizations: a cognitive framework across disciplines," CRPI, vol. 3, no. 1, p. 29, 2018.

[9] E. Dimara, A. Bezerianos, and P. Dragicevic, "Conceptual and Methodological Issues in Evaluating Multidimensional Visualizations for Decision Support," IEEE TVCG, vol. 24, no. 1, pp. 749759, 2018.
[10] T. Asahi, D. Turo, and B. Shneiderman, "Using Treemaps to Visualize the Analytic Hierarchy Process," Inf. Syst. Res., vol. 6, no. 4, pp. 357-375, 1995.

[11] C. Williamson and B. Shneiderman, "The dynamic HomeFinder: evaluating dynamic queries in a real-estate information exploration system," in ACM IGIR, 1992, pp. 338-346.

[12] G. Carenini and J. Loyd, "ValueCharts," in ACM AVI, 2004, p. 150.

[13] S. Gratzl, A. Lex, N. Gehlenborg, H. Pfister, and M. Streit, "LineUp: Visual Analysis of Multi-Attribute Rankings," IEEE TVCG, vol. 19, no. 12, pp. 2277-2286, 2013.

[14] S. Pajer, M. Streit, T. Torsney-Weir, F. Spechtenhauser, T. Muller, and H. Piringer, "WeightLifter: Visual Weight Space Exploration for Multi-Criteria Decision Making," IEEE TVCG, vol. 23, no. 1, pp. 611-620, 2017.

[15] Y. Ahn and Y.-R. Lin, "FairSight: Visual Analytics for Fairness in Decision Making," IEEE TVCG, pp. 1-1, 2019.

[16] S. Afzal, R. Maciejewski, and D. S. Ebert, "Visual analytics decision support environment for epidemic modeling and response evaluation," in IEEE VAST, 2011, pp. 191-200.

[17] A. Savikhin, Hon Cheong Lam, B. Fisher, and D. S. Ebert, "An Experimental Study of Financial Portfolio Selection with Visual Analytics for Decision Support," in IEEE HICSS, 2011, pp. 1-10.

[18] N. Ferreira, M. Lage, H. Doraiswamy, H. Vo, L. Wilson, H. Werner, M. Park, and C. Silva, "Urbane: A 3D framework to support data driven decision making in urban development," in IEEE VAST, 2015, pp. 97-104.

[19] E. Kandogan, A. Balakrishnan, E. M. Haber, and J. S. Pierce, "From Data to Insight: Work Practices of Analysts in the Enterprise," IEEE CGEA, vol. 34, no. 5, pp. 42-50, 2014.

[20] E. Dimara, A. Bezerianos, and P. Dragicevic, "Narratives in Crowdsourced Evaluation of Visualizations: A Double-Edged Sword?" in ACM CHI, 2017, pp. 5475-5484.

[21] J. Hullman, X. Qiao, M. Correll, A. Kale, and M. Kay, "In Pursuit of Error: A Survey of Uncertainty Visualization Evaluation," IEEE TVCG, vol. 25, no. 1, pp. 903-913, 2019.

[22] Y.-S. Kim, L. A. Walls, P. Krafft, and J. Hullman, "A Bayesian Cognition Approach to Improve Data Visualization," in ACM CHI, 2019, pp. 1-14.

[23] E. Dimara, S. Franconeri, C. Plaisant, A. Bezerianos, and P. Dragicevic, "A Task-Based Taxonomy of Cognitive Biases for Information Visualization," IEEE TVCG, vol. 26, no. 2, pp. 1413-1432, 2020.

[24] L. M. Padilla, S. C. Castro, P. S. Quinan, I. T. Ruginski, and S. H. Creem-Regehr, "Toward Objective Evaluation of Working Memory in Visualizations: A Case Study Using Pupillometry and a DualTask Paradigm," IEEE TVCG, vol. 26, no. 1, pp. 332-342, 2020.

[25] C. Kinkeldey, A. M. MacEachren, M. Riveiro, and J. Schiewe, "Evaluating the effect of visually represented geodata uncertainty on decision-making: systematic review, lessons learned, and recommendations," Cartogr Geogr Inf Sci, vol. 44, no. 1, pp. 1-21, 2017.

[26] J. S. B. T. Evans and K. E. Stanovich, "Dual-Process Theories of Higher Cognition: Advancing the Debate," Perspect Psychol Sci, vol. 8 , no. 3, pp. 223-241, 2013.

[27] M. Sedlmair, P. Isenberg, D. Baur, and A. Butz, "Evaluating information visualization in large companies," in BELIV Workshop, 2010, pp. 79-86.

[28] S. Kandel, A. Paepcke, J. M. Hellerstein, and J. Heer, "Enterprise Data Analysis and Visualization: An Interview Study," IEEE TVCG, vol. 18, no. 12, pp. 2917-2926, 2012.

[29] M. Kim, T. Zimmermann, R. DeLine, and A. Begel, "The emerging role of data scientists on software development teams," in ICSE, 2016, pp. 96-107.

[30] M. Behrisch, D. Streeb, F. Stoffel, D. Seebacher, B. Matejek, S. H. Weber, S. Mittelstadt, H. Pfister, and D. Keim, "Commercial Visual Analytics Systems-Advances in the Big Data Analytics Field," IEEE TVCG, vol. 25, no. 10, pp. 3011-3031, 2019.

[31] A. Mosca, S. Robinson, M. Clarke, R. Redelmeier, S. Coates, D. Cashman, and R. Chang, "Defining an Analysis: A Study of Client-Facing Data Scientists," in EuroVis, 2019.

[32] S. Alspaugh, N. Zokaei, A. Liu, C. Jin, and M. A. Hearst, "Futzing and Moseying: Interviews with Professional Data Analysts on Exploration Practices," IEEE TVCG, vol. 25, no. 1, pp. 22-31, 2019.

[33] A. Batch and N. Elmqvist, "The Interactive Visualization Gap in Initial Exploratory Data Analysis," IEEE TVCG, vol. 24, no. 1, pp. 278-287, 2018.

[34] K. Madanagopal, E. D. Ragan, and P. Benjamin, "Analytic Provenance in Practice: The Role of Provenance in Real-World Visualiza- 
tion and Data Analysis Environments," IEEE CGEA, vol. 39, no. 6, pp. 30-45, 2019.

[35] N. Boukhelifa, M.-E. Perrin, S. Huron, and J. Eagan, "How Data Workers Cope with Uncertainty," in ACM CHI, 2017, pp. 36453656.

[36] D. Fisher, R. DeLine, M. Czerwinski, and S. Drucker, "Interactions with big data analytics," interactions, vol. 19, no. 3, p. 50, 2012.

[37] D. M. Russell, "Simple is Good: : Observations of Visualization Use Amongst the Big Data Digerati," in ACM AVI, 2016, pp. 7-12.

[38] A. Sarikaya, M. Correll, L. Bartram, M. Tory, and D. Fisher, "What Do We Talk About When We Talk About Dashboards?" IEEE TVCG, vol. 25, no. 1, pp. 682-692, 2019.

[39] H. Simon, "Administrative behavior: a study of decision-making processes in administrative organization." 1947.

[40] J. G. March and H. A. Simon, "organizations." 1958.

[41] G. P. Huber and R. R. McDaniel, "The decision-making paradigm of organizational design," Manage Sci, vol. 32, no. 5, pp. 572-589, 1986.

[42] J. W. Rivkin and N. Siggelkow, "Balancing search and stability: Interdependencies among elements of organizational design," Manage Sci, vol. 49, no. 3, pp. 290-311, 2003.

[43] J. Clement and P. Puranam, "Searching for structure: Formal organization design as a guide to network evolution," Manage Sci, vol. 64, no. 8, pp. 3879-3895, 2018.

[44] W. Ocasio and H. Kim, "The circulation of corporate control: Selection of functional backgrounds of new ceos in large us manufacturing firms, 1981-1992," Adm. Sci. Q., vol. 44, no. 3, pp. 532-562, 1999.

[45] E. Dane and M. G. Pratt, "Exploring intuition and its role in managerial decision making," Acad Manage Rev, vol. 32, no. 1, pp. 33-54, 2007.

[46] P. R. Laughlin and J. Adamopoulos, "Social combination processes and individual learning for six-person cooperative groups on an intellective task." J Pers Soc Psychol, vol. 38, no. 6, p. 941, 1980.

[47] K. Weber and D. Waeger, "Organizations as polities: An open systems perspective," Acad. Manag. Ann., vol. 11, no. 2, pp. 886918, 2017.

[48] W. Ocasio, "Towards an attention-based view of the firm," Strateg. Manag. J., vol. 18, no. S1, pp. 187-206, 1997.

[49] J. Pfeffer and G. R. Salancik, "Organizational decision making as a political process: The case of a university budget," Adm. Sci. Q., pp. 135-151, 1974.

[50] R. M. Cyert and J. G. March, "A behavioral theory of the firm," Englewood Cliffs, NJ, vol. 2, no. 4, pp. 169-187, 1963.

[51] R. R. Nelson and S. G. Winter, "An evolutionary theory of economic change," Tech. Rep., 1982

[52] J. W. Meyer and B. Rowan, "Institutionalized organizations: Formal structure as myth and ceremony," Am. J. Sociol, vol. 83, no. 2, pp. 340-363, 1977.

[53] P. J. DiMaggio and W. W. Powell, "The iron cage revisited: Institutional isomorphism and collective rationality in organizational fields," Am. Sociol. Rev., pp. 147-160, 1983.

[54] M. D. Cohen, J. G. March, and J. P. Olsen, "A garbage can model of organizational choice," Adm. Sci. Q., pp. 1-25, 1972.

[55] A. L. Kalleberg and M. E. Van Buren, "Is bigger better? explaining the relationship between organization size and job rewards," Am. Sociol. Rev., pp. 47-66, 1996.

[56] P. R. Sparrow and D. Davies, "Effects of age, tenure, training, and job complexity on technical performance." Psychol Aging, vol. 3, no. 3, p. 307, 1988 .

[57] H. A. Simon, "The new science of management decision." 1960.

[58] A. Newell, H. A. Simon, and Others, Human problem solving. Prentice-Hall Englewood Cliffs, NJ, 1972, vol. 104, no. 9.

[59] J. R. Wood and L. E. Wood, "Card sorting: current practices and beyond," Journal of Usability Studies, vol. 4, no. 1, pp. 1-6, 2008.

[60] J. P. Cornelissen and M. D. Werner, "Putting framing in perspective: A review of framing and frame analysis across the management and organizational literature," Acad. Manag. Ann., vol. 8, no. 1, pp. 181-235, 2014.

[61] N. Fligstein, "Social skill and institutional theory," Am Behav Sci, vol. 40, no. 4, pp. 397-405, 1997.

[62] B. Crandall, G. Klein, and R. R. Hoffman, Working Minds:A Practitioner's Guide to Cognitive Task Analysis. The MIT Press, 2006.

[63] Zhicheng Liu and J. T. Stasko, "Mental Models, Visual Reasoning and Interaction in Information Visualization: A Top-down Perspective," IEEE TVCG, vol. 16, no. 6, pp. 999-1008, 2010.
[64] V. Braun and V. Clarke, "Using thematic analysis in psychology," Qual. Res. Psychol., vol. 3, no. 2, pp. 77-101, 2006.

[65] M. J. Eppler and J. Mengis, "The concept of information overload: A review of literature from organization science, accounting, marketing, mis, and related disciplines," Inf. Soc., vol. 20, no. 5, pp. 325-344, 2004.

[66] J. Joseph and W. Ocasio, "Architecture, attention, and adaptation in the multibusiness firm: General electric from 1951 to 2001," Strateg. Manag. J., vol. 33, no. 6, pp. 633-660, 2012.

[67] H. Guetzkow and H. A. Simon, "The impact of certain communication nets upon organization and performance in task-oriented groups," Manage Sci, vol. 1, no. 3-4, pp. 233-250, 1955.

[68] R. P. Beatty and E. J. Zajac, "Managerial incentives, monitoring, and risk bearing: A study of executive compensation, ownership, and board structure in initial public offerings," Adm. Sci. Q., pp. 313-335, 1994

[69] A. D. Brown and K. Starkey, "The effect of organizational culture on communication and information," J. Manag. Stud, vol. 31, no. 6, pp. $807-828,1994$.

[70] C. W. Choo, P. Bergeron, B. Detlor, and L. Heaton, "Information culture and information use: An exploratory study of three organizations," J Am Soc Inf Sci Tec, vol. 59, no. 5, pp. 792-804, 2008.

[71] E. Dimara and C. Perin, "What is Interaction for Data Visualization?" IEEE TVCG, vol. 26, no. 1, pp. 119-129, 2020.

[72] P. Isenberg, N. Elmqvist, J. Scholtz, D. Cernea, Kwan-Liu Ma, and H. Hagen, "Collaborative visualization: Definition, challenges, and research agenda," Inf. Vis, vol. 10, no. 4, pp. 310-326, 2011.

[73] Z. Zhang, B. Wang, F. Ahmed, I. Ramakrishnan, R. Zhao, A. Viccellio, and K. Mueller, "The five ws for information visualization with application to healthcare informatics," IEEE TVCG, vol. 19, no. 11, pp. 1895-1910, 2013.

[74] H. Xia, N. Henry Riche, F. Chevalier, B. De Araujo, and D. Wigdor, "Dataink: Direct and creative data-oriented drawing," in ACM CHI, 2018, pp. 1-13.

[75] W. Willett, Y. Jansen, and P. Dragicevic, "Embedded data representations," IEEE TVCG, vol. 23, no. 1, pp. 461-470, 2016.

[76] V. Setlur, S. E. Battersby, M. Tory, R. Gossweiler, and A. X. Chang, "Eviza: A natural language interface for visual analysis," in ACM UIST, 2016, pp. 365-377.

[77] B. A. Aseniero, T. Wun, D. Ledo, G. Ruhe, A. Tang, and S. Carpendale, "STRATOS: Using Visualization to Support Decisions in Strategic Software Release Planning," in ACM CHI, 2015, pp. 14791488 .

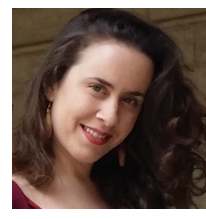

Evanthia Dimara Evanthia Dimara is an Assistant Professor at Utrecht University. Her fields of research are Data Visualization and HumanComputer Interaction. Her focus is on decision making - how to help people make unbiased and informed decisions alone or in groups.

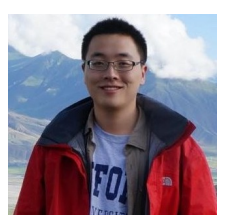

Harry Zhang Harry Zhang is a Ph.D. candidate at Northwestern University. He studies organization theory, particularly how rules, institutions and culture in organizations affect the experience of their members and organizational behaviors.

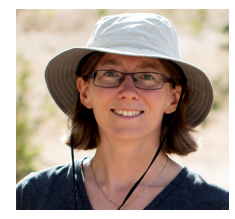

Melanie Tory Melanie Tory is a user research manager at Tableau focusing on interactive visual data analysis. Her team's research explores how people engage with analytic tools to ask and answer questions with data and how we can better design tools to support this process.

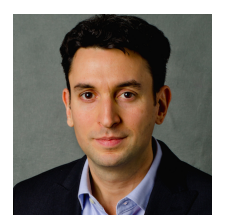

Steven Franconeri Steven Franconeri is a Professor of Psychology at Northwestern University and Director of the Northwestern Cognitive Science Program. He studies visuospatial thinking and visual communication, across psychology, education, and information visualization. 